\title{
Simulation and experimental validation of vibro-acoustic transmission through a passive automotive door mount system
}

\author{
D. A. Desai \\ Tshwane University of Technology, Pretoria, South Africa
}

\begin{abstract}
Automotive manufacturers are faced with many challenges to meet everincreasing industry standards and satisfy customer demands. Two such requirements are the growing demand for reliable automotive products that can withstand severe dynamic environments as well as the reduction of automotive interior noise levels, which is an important concern in noise, vibration and harshness (NVH) design because of its huge effect as a marketing tool and on the general pleasure of driving. However, anecdotal evidence suggests that, until now, the characterisation of structure-borne door panel vibration and its subsequent noise emission characteristics in terms of its door mount system has not yet been contemplated. Thus, a three-dimensional finite element (FE) model with full coupling between the passenger cabin and vehicle body, to simulate the complex, transient door panel vibration and internal acoustic wave propagation as a function of the door mount system, when provoked by an external impact force, is proposed. With reference to a real door mount system, a discrete, multidegree-of-freedom, time domain numerical model is developed and solved using ABAQUS ${ }^{\circledR}$ FE code. An objective function value based on the sound pressure level transmitted to the driver's head position is then computed in order to evaluate the performance of the door mount system. An experimental campaign was thereafter undertaken to validate the numerical model based on time response data. The measured data were compared with the predicted values, the results of which exhibited good correlation.
\end{abstract}

Keywords: automotive wheel impact analysis, $N V H$, automotive interior noise. 


\section{Introduction}

The automobile has become an integral part of our daily lives. As a result, stricter standards for noise emissions are leading the automotive industry to go beyond what has been achieved in the past. Consequently, automotive interior noise levels and their respective acoustic signatures (the way the vehicle sounds to its occupants under various operating conditions) are becoming competitive differentiators for automakers.

One of the main sources of interior noise in modern passenger vehicles is structure-borne noise generated by road-tyre interaction, especially at higher speeds on rough road surfaces [1]. The spectrum of road noise has been found to be quite strongly influenced by the vibration characteristics of the car body, in the range of the first few acoustic cavity resonances. Furthermore, studies on impact transient dynamics of acoustic-structural systems are essential for understanding and evaluating the acoustic performance of transportation systems where the dynamic response is fundamental to sustainable and satisfactory operation. Structural vibrations of the car body subjected to impact loads due to potholes or obstacles result in further vibrations of the door panels, which enclose the passenger compartment. As a result; in addition to the "shock" wave generated, bending waves progress, that bring air in motion causing radiation of sound. These vibrating panels radiate noise to the interior of the compartment where the noise can be either amplified or reduced by the cavity's acoustic characteristics. Testing has shown a strong correlation between panel motion and measured noise. Jennequin [2] has indicated that the nature of noise inside a vehicle is complex, because it is determined inter alia by the behaviour and interaction of the vehicle structure, the vibrating door panels and the air cavity within the vehicle itself. The passenger cabin of a vehicle can be considered to be an irregular shaped acoustic field enclosed by body panels, thereby making the interpretation of the acoustic field expensive. Since the passenger cabin of a vehicle forms a cavity, resonant conditions can develop, characterised by acoustic modes of vibration.

According to Hörnlund and Papazoglu [3], vehicle doors play an important part when performing structure-borne road noise simulations since they comprise large surfaces, which exert a significant impact on the interior cabin pressure when in motion. Hence, an increasingly greater challenge facing the automotive manufacturer is the prediction of cabin vibro-acoustic behaviour, when subjected to impact loading, which is strongly related to the vibrational motion of the door panels that are connected to the vehicle structure through passive door mounts, door seals, latch and striker mechanisms.

Although studies on the estimation of sound radiation from vibrating plates with classical boundary conditions have not escaped the attention of researchers, no comparable efforts have been made using non-classical (complex) boundary conditions, such as automotive door mounts. According to Desai et al. [4], door mount systems merit study because anecdotal evidence suggests that the effects of non-classical panel support or fixing conditions in terms of noise generation have not yet been contemplated or adequately studied within a vibro-acoustic 
setting. Many studies on panel contributions assume rigid boundary conditions and neglect the actual modelling of the panel support conditions that could otherwise have an effect on the panel stiffness, which, in turn, could alter the behaviour of the door panel itself. In an excellent study; Nefske et al. [5] showed that boundary conditions could shift the frequencies of the acoustic modes, move nodal lines and produce important changes in the overall noise response. Viewed by many as the next step in the evolution of vibro-acoustics, these conclusions fascinated many noted researchers at that time. According to Rivin [6], classical support conditions are often oversimplified in theory, in that complicating factors, such as friction and clearance in the connection, are not considered, as they are difficult to identify in practice.

The establishment of cabin behavioural trends in the early stages of vehicle body development is highly recommended, since it is costly to introduce changes after body manufacture. Also, because the time to market is constantly decreasing while the pressure to save costs are increasing, $\mathrm{NVH}$ engineers seek to define and optimize a vehicle's vibrational and acoustic behaviour as early as possible. However, such behaviour can be predicted using computer-based models employing the finite element method [4]. The development of such predictive models is therefore an effective and sensible approach as the flexibility of such a model can provide a powerful tool for in depth investigations without the problems often correlated with trial and error methods.

However, the phenomenon of door panel vibration and cabin acoustics is complex as it comprises the simultaneous interaction of many competing factors. Some of the complexities are introduced by the dynamics of structural joints, the multi-body physics involved, the fluid-structure interaction between structural panels and interior acoustics, the damping characteristics, door seal hyperelasticity and non-linear dynamic behaviours. Such issues cannot be resolved by simply using finer finite element meshes, but require the development of specific models that incorporate design parameters affecting interior sound spectra [7]. The development of such predictive models therefore necessitates an intensive study of the door mount system together with the coupling phenomenon between the passenger cabin and the vehicle body structure.

In this work, the ABAQUS/Explicit finite element code has been employed to characterise the vibro-acoustic behaviour of a typical door mount, welded to a simplified, scaled-down vehicle model. Actual in-service conditions in terms of impact loading, contact, friction and damping phenomena have been considered. The validity of the developed numerical model is also experimentally verified based on time response functions, the results of which are presented in this paper. Comparative analyses between the simulated and experimental results show good agreement. This proves that the developed numerical model is functional and reliable. The insights gained into the noise generation and transmission behaviour of the system under investigation may be materialized into realistic design modifications and can augment existing $\mathrm{NVH}$ studies or algorithms enabling $\mathrm{NVH}$ engineers to move closer towards cabin acoustic refinement in pursuit of their quest for quieter passenger cabins. 


\section{Geometry and model definition}

An acoustic-structural interaction model is developed and used to conduct a mixed noise-vibration analysis in order to represent the coupled vibration and acoustic phenomena. The vibro-acoustical coupling is described in terms of physical degrees of freedom rather than the conventional modal analysis approach because the modal density of such vibro-acoustical models are generally very high that classical modal analysis may not be applicable. Also, the acoustic domain must often be discretized by very fine finite element mesh, thus resulting in very large and dense systems that must be solved by specific procedures. Since the study entails a short-duration, transient vibro-acoustic dynamic analysis involving wave propagation; that is, the important dynamics occur at a time scale that is short compared to the wave speed of the solid medium and acoustic wave speed of the fluid, the ABAQUS/Explicit solver, employing the central difference rule, to integrate the equations of motion explicitly through time, is employed. The dynamic equilibrium defined by eqn (1) is solved continuously at the beginning of each increment.

In order to minimise computational efforts and costs, a simplified car body of approximately one-third scale, stripped of its internal trimming but with its door mounts, door seal, latch and striker mechanism remaining as in production, is investigated. A stiff vehicle structure was developed using dimensional analysis and similitude theory in order to maintain approximate relative occurrence on par with a typical full-size real vehicle structure. The case under study consist essentially of a vehicle's front right-hand door panel with its respective upper and lower door mount assemblies, a door seal and a vehicle body structure constituting an enclosed passenger cabin, without any noise packaging treatment. The door is articulated by means of the mount assemblies connected to the Apillar and door, respectively. The test structure is constructed from both $25 \mathrm{~mm}$ square and $100 \mathrm{~mm} \times 50 \mathrm{~mm}$ mild steel tubing (A-pillar); clad by $3 \mathrm{~mm}$ thick mild steel plates. The interior of the passenger cabin is sealed by means of a door seal at the front right-hand door. The test structure under consideration is shown in fig. 1 , the components of which are representative of a real system.
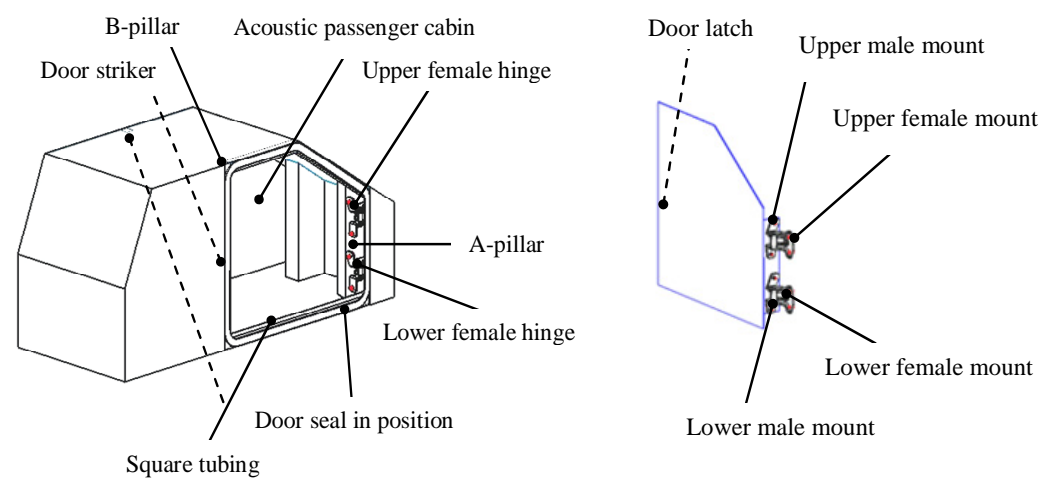

Figure 1: $\quad$ Simplified vehicle with actual door mount system. 


\section{Mathematical models}

\subsection{Dynamic equilibrium}

The dynamic equilibrium equation that must be solved at the beginning of each time increment $t$, may be defined as

$$
\mathbf{M} \ddot{\mathbf{u}}_{(t)}=\mathbf{P}_{(t)}-\mathbf{I}_{(t)}
$$

where $\mathbf{M}$ is the nodal mass matrix, $\ddot{\mathbf{u}}$ represents the nodal accelerations, $\mathbf{P}$ is the externally applied impact force and $\mathbf{I}$ the internal element forces.

\subsection{Fluid behaviour}

The governing equilibrium equation for small motions of a compressible, adiabatic, inviscid fluid with a finite wave speed is taken to be

$$
\frac{\partial p}{\partial \mathbf{x}}+r \dot{\mathbf{u}}^{f}+\rho^{f} \ddot{\mathbf{u}}^{f}=0
$$

where $p$ is the acoustic pressure in the fluid, $\dot{\mathbf{u}}^{f}$ is the fluid particle velocity, $\ddot{\mathbf{u}}^{f}$ is the fluid particle acceleration, $\rho^{f}$ is the density of the fluid and $r$ is the volumetric drag. This equation is modelled using a pressure-based finite element formulation with nodal pressures on the finite element mesh. Furthermore, since the constitutive behaviour of the fluid is assumed to be inviscid and compressible

$$
p=-K_{f} \frac{\partial}{\partial \mathbf{x}} \cdot \mathbf{u}^{f}
$$

where $K_{f}$ is the bulk modulus of the fluid, $\mathbf{x}$ the spatial position, and $\mathbf{u}^{f}$ represents the fluid particle displacement. Hence, the fluid, which serves as an additional mechanical load on the inner body panels, is treated as an elastic solid with a finite bulk modulus and a negligible shear modulus. The sound pressure level (SPL) at a particular location $p_{\mathrm{i}}$ may be defined as

$$
p_{\mathrm{i}}=20 \log _{10}\left(\frac{\mathrm{P} / \sqrt{2}}{P_{\text {ref }}}\right)
$$

where $P$ is the pressure and $P_{r e f}$ is the standard reference pressure of $2 \times 10^{-5} \mathrm{~Pa}$.

\subsection{Hyper-elastic door seal model}

In this work, the popular EPDM sponge-dense, elastomeric door seal is used. The door seal is modelled with the hyper-elastic material model, which is a nonlinear, continuum model. This model incorporates the highly compressible nature of EPDM sponge door seals and is characterised by the Marlow strain 
energy potential described by Morman [8] and applied by Wagner et al. [9]. The Marlow strain energy potential may be expressed in the form

$$
U=U_{d e v}\left(\bar{I}_{1}\right)+U_{v o l}\left(J_{e l}\right)
$$

where $U$ is the strain energy per unit of reference volume with $U_{d e v}$ as its deviatoric part, $U_{v o l}$ as its volumetric part and $J_{e l}$ represents the elastic volume ratio. The deviatoric part of the strain energy potential $\bar{I}_{1}$ is defined by providing biaxial test data. A 91\% seal compression, similar to that used by Stenti et al. [10], which represents the door seal compression level of a typical sedan vehicle, was used in the case under investigation.

\subsection{Energy dissipation model}

According to popular belief and experience, the determination of damping characteristics in complex structures is a very difficult process. Therefore, an approximate damping scheme should be employed. According to Cook et al. [11], experimental observations of the vibratory response of coupled structure could be used to assign a single damping fraction to the entire structure. Hence, the popular Rayleigh spectral damping scheme described by Zienkiewicz and Taylor [12] was employed in this study and may be defined as:

$$
C_{i}=\alpha_{i} M_{i}+\beta_{i} K_{i}
$$

where $\boldsymbol{C}$ is the Rayleigh damping matrix, $\alpha$ and $\beta$ are called the mass and stiffness proportional damping constants; respectively, $K$ the stiffness matrix and the subscript ${ }_{i}$ refers to the mode number. However, for a given mode ${ }_{i}$, the fraction value of critical damping is

$$
\xi_{i}=\frac{\alpha_{i}}{2 \omega_{i}}+\frac{\beta_{i} \omega_{i}}{2}
$$

where $\omega_{i}$ is the natural frequency (radians per unit time) at this mode and $\xi_{i}$ the damping ratio for ${ }_{i}$ th mode. By careful analysis of the theory presented by Cook et al. [11] and Clough and Penzien [13], it seems possible that the two Rayleigh damping factors $\alpha$ and $\beta$ can be evaluated by experimentally determining the fractions of critical damping $\left(\xi_{1}\right.$ and $\left.\xi_{2}\right)$ at two successive frequencies $\left(\omega_{1}\right.$ and $\left.\omega_{2}\right)$ and then solving eqn (7) simultaneously.

\section{Mathematical formulation}

\subsection{Basic governing equation}

The vehicle structure and its respective components are modelled as deformable bodies comprising flexible wall panels enclosing an acoustic cavity. The fullycoupled fluid-structure interaction system may be represented in a finite element type discretization matrix form by 


$$
\left[\begin{array}{cc}
M_{s} & 0 \\
A & M_{f}
\end{array}\right]\left\{\begin{array}{l}
\ddot{u}_{s} \\
\ddot{p}_{f}
\end{array}\right\}+\left[\begin{array}{cc}
C_{s} & 0 \\
0 & C_{f}
\end{array}\right]\left\{\begin{array}{l}
\dot{u}_{s} \\
\dot{p}_{f}
\end{array}\right\}+\left[\begin{array}{cc}
K_{s} & -A^{T} \\
0 & K_{f}
\end{array}\right]\left\{\begin{array}{c}
u_{s} \\
p_{f}
\end{array}\right\}=\left\{\begin{array}{c}
f_{s} \\
0
\end{array}\right\}
$$

where $\mathbf{M}, \mathbf{C}$ and $\mathbf{K}$ are the mass, damping and stiffness matrices with the subscripts $s$ and $f$ representing the structure and fluid medium, respectively. $\mathbf{p}_{f}$ is the acoustic pressure with $\ddot{\mathbf{p}}_{f}$ and $\dot{\mathbf{p}}_{f}$ its second and first derivatives, respectively, at the grid points or nodes of the finite element model, which discretizes the air volume of the passenger cabin. The non-symmetric matrix $\mathbf{A}$ represents the coupling between the structure and the cavity and $f_{s}$ is the external impact force vector.

\subsection{Boundary conditions}

In this study, the dynamics of the vehicle structure including its door mount system is analysed under free-free boundary conditions. The welded mount connections are specified as zero-value boundary conditions and were modelled as a fixed connection. Since the acoustic medium is adjacent to the vehicle structure, acoustic-structural coupling occurs. The pressure field creates a normal surface traction on the structure and the acceleration field creates the natural forcing term at the fluid boundary. At the acoustic-structural boundary where the motion of the acoustic medium is directly coupled to the motion of the solid structure, the acoustic and structural media have the same acceleration normal to the boundary, so that

$$
\mathbf{n} \cdot \ddot{\mathbf{u}}^{f}=\mathbf{n} \cdot \ddot{\mathbf{u}}^{s}
$$

where the vector $\mathbf{n}$ represents the inward normal to the acoustic medium at the boundary.

\subsection{Definition of external impact load}

Experimental road-load measurements performed by Rupp and Grubisic [14] on vehicles driven on a potholed road surface suggested typical wheel suspension input forces of around $10000 \mathrm{~N}$. In order to preserve dynamic similarity to an actual full-scale vehicle, application of the relevant scaling laws suggests that the model under investigation be excited by a $1000 \mathrm{~N}$ impact force. This vertical force was applied for a period of 1 milli-second at the front right-hand suspension point.

\section{Numerical modelling}

\subsection{Mesh design}

Although the meshes at the acoustic-structural tied boundary may be nodally nonconforming, mesh refinement, which depends on wave speeds in the two media, affects the accuracy of the solution. The mesh for the medium with the 
lower wave speed (air) should generally be more refined and therefore should be the slave surface. Since material properties affect mesh parameters for wave propagation problems and hence affect the accuracy of the solution, the discretization protocol of the FE method require at least six nodes per wavelength [7]. The finite element model was created using a wide variety of appropriate finite elements and material properties in order to represent the behaviour of the various interacting bodies, adequately. The model essentially contains a mix of first and second-order structural elements to model the vehicle structure and first-order acoustic elements to model the passenger cabin (due to software limitations). Adaptive meshing was defined at and around the impact zone. All meshes were check in terms of standard mesh quality protocols.

\subsection{Contact interactions}

The finite element simulation models Mohr-Coulomb type friction between all relevant contacting surfaces. Deformable-to-deformable, small-sliding contact conditions are imposed on the model where applicable.

\section{Experimental characterisation}

\subsection{General test set-up}

The test vehicle was supported using four soft elastic bands in an overhead fashion to simulate the free-free boundary condition used in the dynamics calculations and was fabricated and assembled in a similar way to that of an actual sedan vehicle. All air gaps in the vehicle body were sealed with highquality non-porous duct tape in order to minimise acoustic losses.

\subsection{Modal analysis}

In order to facilitate the solution of eqns $(6,7)$ an experimental modal analysis was carried out using the single reference testing method. A miniature Dytran 3023M2 tri-axial accelerometer of mass 3 grams was employed in conjunction with standard equipment comprising a PCB 086 C03 modal hammer linked to a four-channel DSP SigLab spectrum analyser via Piezotronics PCB 480E09 signal conditioning units. A PC was used for collecting and managing the acquired measurements.

A total of 113 equally distributed excitation points have been selected with a spatial resolution of $80 \mathrm{~mm}$ in the three global directions, resulting in a well defined experimental model. Since closely spaced modes were expected, the required modal parameters for eqn (7) were estimated from 339 measured frequency response function (FRF) accelerance spectra (113 points $\times 3$ global directions) using the Global-M frequency domain curve-fitting algorithm found in the MODENT modal-analysis software package. 


\subsection{Transient impact response analysis}

A transient vibro-acoustic dynamic analysis was carried out in order to measure the door panel vibration and interior SPL at the driver's ear position. The interior SPL and door panel acceleration were measured with a precision TMS 130P10 1/4-inch condenser microphone and the Dytran accelerometer described in paragraph 6.2 respectively, linked to the SigLab FFT analyser via the two PCB signal conditioning units described above.

The analyser and a shaker were linked to a MB Dynamics SS250 amplifier, all of which were located outside the vehicle to reduce the effects of reflection from the body of the instruments, thus improving the accuracy of the measurements. The MB Dynamics M50A electromagnetic shaker representing the road suspension input was carefully aligned and connected to the front righthand vehicle floor panel via a $5 \mathrm{~mm}$ diameter, $10 \mathrm{~mm}$ long steel threaded stinger coincident with that of the finite element model.

A PCB 208 C03 force transducer was stud-mounted to the vehicle suspension point via a small aluminium mounting block in the force transmission path and connected to the shaker by means of the stinger. The shaker was bolted to a rigid table to enable the reaction force to be absorbed and not transferred to the structure under test. The experimental set-ups are depicted in figs. 2 and 3. Note that the bolts shown in fig. 3 were omitted in the actual test.
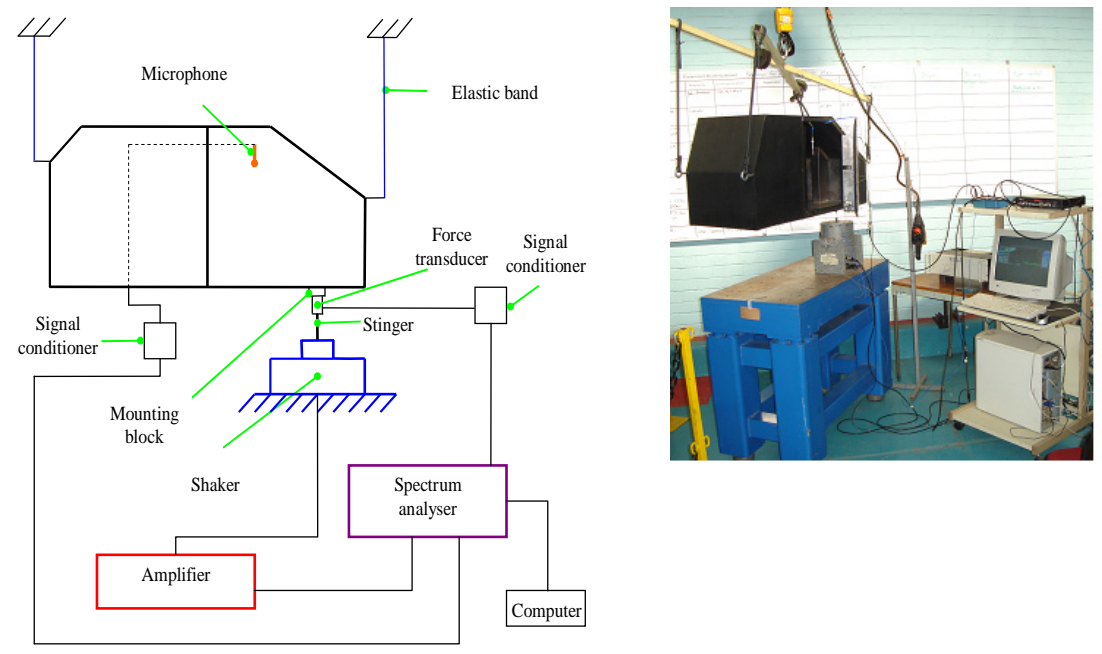

Figure 2: $\quad$ Experimental set-up for measurement of interior SPL. 


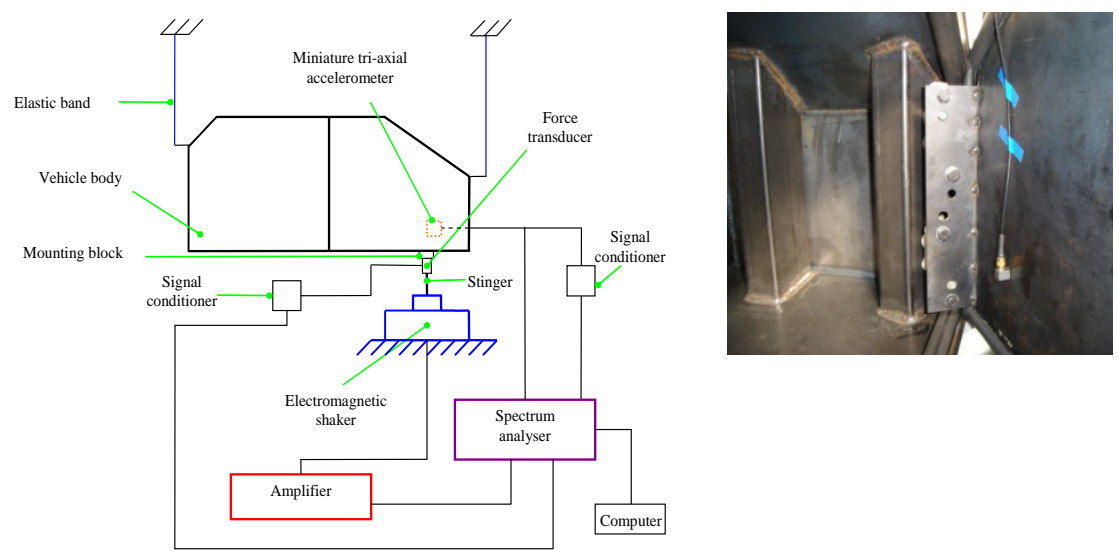

Figure 3: $\quad$ Experimental set-up for measurement of door panel vibration.

\section{Results}

The first two experimentally-determined damping ratios with their corresponding natural frequencies were $3.3 \%(97.85 \mathrm{~Hz})$ and $3.2 \%(106.78 \mathrm{~Hz})$, respectively and were subsequently used to solve eqn (7). With a model size of approximately $1 / 2$ million degrees-of-freedom (including contact elements and Lagrange multiplier variables), the simulation converged at 80.17 hours of CPU time in approximately 2 million increments. The analysis was executed on a $2.53 \mathrm{GHz}$ dual core notebook with $3 \mathrm{~GB}$ of RAM, running on a Windows XP operating system. At the early stages of the analysis the time increments were very small because the initial impact initiates stress wave effects, which propagate throughout the model, carrying energy with them. Hence, small increments are required to model the dynamics accurately during this period.

The left-hand plot in fig. 4 depicts the evolution and correlation between the predicted and measured values of the objective function, which is defined as the SPL at the driver's head location described by eqn (4). The front right-hand door panel inertance is shown in the right-hand plot.
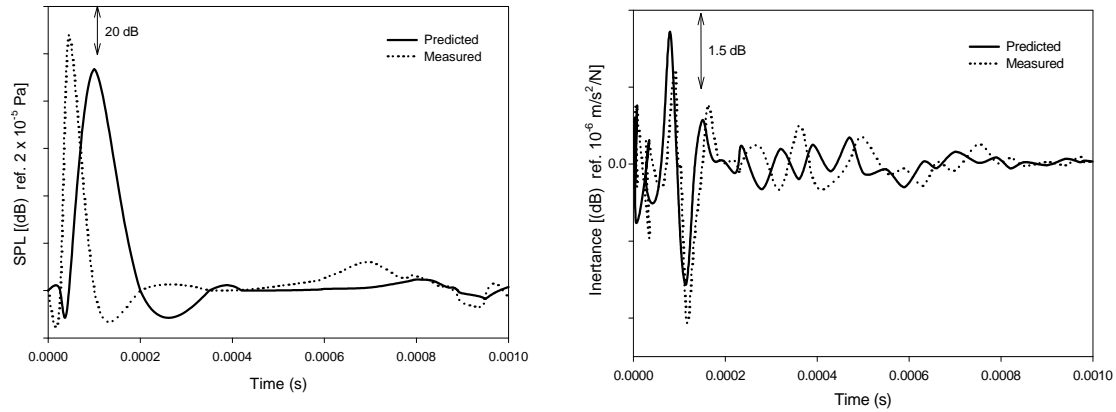

Figure 4: Vibro-acoustic signatures. 


\section{Discussion}

The correlation between the analysis and experimental results are quite encouraging where it is evident that the overall characteristics are similar to each other, despite the complexity of the structure under investigation. Differences may be attributed to the difficulty in the estimation of damping, noise within the measuring equipment, limitations in the numerical procedure and physical measurement process, neglecting stiffness residue, pre-strain and panel curvature effects due to welding of the test structure that have not been explicitly modelled. Compared to real vehicles, the SPLs in fig. 4 may seem high at first sight. However, this difference is primarily due to the effect of scaling applied to the vehicle model.

\section{Conclusion}

A computational procedure for predicting vibro-acoustic transmission through a passive door mount system has been proposed and demonstrated by FE analysis. Correlation between the predicted and measured signatures showed good agreement. It is hoped that this research, which has been undertaken in collaboration with Ford Motor Company of SA, will make deeper inroads into how door mount systems behave in a vibro-acoustic setting by filling the identified gap in knowledge and by promoting a greater understanding. This will allow for specific vibro-acoustic characteristics to be engineered at the body-inwhite stage of vehicle development.

\section{References}

[1] Dehandschutter, W., Sas, P. and Banfo, G.L., Active control of rolling noise in a passenger car through structural and acoustic control. In: SAS, P. (ed.). Proceedings of ISMA21, 1996, International Conference on Noise and Vibration Engineering, Vol. 1, Katholieke Universiteit, Leuven, Belgium. PMA: 401- 412, 1996.

[2] Jennequin, G., Is the computation of noise level inside a car feasible? In: Symposium of Vibration and Noise in Motor Vehicles. London: s.n.: 143137, 1971.

[3] Hörnlund, M. and Papazoglu, A., Analysis and measurements of vehicle door structural dynamic response. M.Sc. dissertation, Lund University, Sweden, 2005.

[4] Desai, D.A., Aylward, R.C., Smith, C.J. and Erasmus, J., Prediction of lowfrequency vibration transmission through an automotive door mount system using the finite element method. Journal for New Generation Sciences, 7(2): 75-90, 2009.

[5] Nefske, D.J., Wolf, J.A. and Howell, L.J., Structural-acoustic finite element analysis of the automobile passenger cabin: a review of current practice. Journal of Sound and Vibration, 80(2), Jan.: 247-266, 1982. 
[6] Rivin, E.I., Stiffness and damping in mechanical design. New York: Marcel Dekker, Inc., 1999.

[7] Lim, T.C., Automotive panel noise contribution modelling based on finite element and measured structural-acoustic spectra. Applied Acoustics, 60, pp. 505-519, 2000.

[8] Morman, K.N., Recent applications of ABAQUS to the analysis of automotive rubber components. ABAQUS User's Conf., Milan, Italy, pp. 23-67, 1998.

[9] Wagner, D.A., Morman, K.N., Gur, Y. and Koka, M.R., Non-linear analysis of automotive door weatherstrip seals. Finite Elements in Analysis and Design, 28, pp. 33-40, 1997.

[10] Stenti, A., Moens, D. and Desmet, W., Dynamic modelling of car door weather seals: a first outline. Proc. of ISMA2004 Int. Conf. on Noise and Vibration Engineering, eds. P. Sas and M. de Munck, 3, Katholieke Universiteit: Leuven, Belgium, pp. 1249-1262, 2004.

[11] Cook, R.D., Malkus, D.S. and Plesha, M.E., Concepts and applications of finite element analysis. $3^{\text {rd }}$ ed. Canada: John Wiley and Sons, Inc., 1989.

[12] Zienkiewicz, O.C. and Taylor, R.L., The finite element method. Vol. $1,5^{\text {th }}$ ed. Oxford: Butterworth-Heinemann, 2000.

[13] Clough, R.W. and Penzien, J., Dynamics of structures. $2^{\text {nd }}$ ed. Singapore: McGraw Hill, 1993.

[14] Rupp, A and Grubisic, V., Reliable and efficient measurement of multiaxial in-service loads on cars and commercial vehicles. Reports in Applied Measurement, 10 (1), pp. 13-18, 1996. 